\title{
Review of: "Thrombocytopenia and splenic platelet directed immune responses after intravenous ChAdOx1 nCov-19 administration"
}

Darrell Ricke ${ }^{1}$

1 Massachusetts Institute of Technology

Potential competing interests: The author(s) declared that no potential competing interests exist.

This is a well written article on thrombocytopenia associated with COVID-19 adenovirus vaccine.

The preprint was posted on June 29, 2021. This reviewer kindly requests the authors to consider citing the following articles:

Lozier JN, Csako G, Mondoro TH, Krizek DM, Metzger ME, Costello R, Vostal JG, Rick ME, Donahue RE, Morgan RA. Toxicity of a first-generation adenoviral vector in rhesus macaques. Hum Gene Ther. 13: pp. 113-124 (2002).

Batarseh KI. Venous thromboembolism and thrombocytopenia associated with adenoviral vector-based COVID-19 vaccines: an explanation of the anomaly. BMJ. Published Online April 19,

2021. https://www.bmj.com/content/373/bmj.n961/rr.

Schultz N.H., Sørvoll I.H., Michelsen A.E., Munthe L.A., Lund-Johansen F., Ahlen M.T. Thrombosis and Thrombocytopenia after ChAdOx1 nCoV-19 Vaccination. N Engl J Med. 2021;384(22):2124-2130.

June 3, 2021

Scully M., Singh D., Lown R., Poles A., Solomon T., Levi M. Pathologic Antibodies to Platelet Factor 4 after ChAdOx1 nCoV-19 Vaccination. N Engl J Med. 2021;384(23):2202-2211.

June 10, 2021

This article describes platelet destruction by macrophages; these macrophages can also be referred to as hemophagocytic histiocytes. This reviewer requests the authors consider mentioning this. 